\title{
Computational Cognitive Epigenetics ${ }^{1}$
}

\author{
Aaron Sloman a and Jackie Chappell ${ }^{\mathrm{b}}$
}

\author{
Schools of Computer Science ${ }^{a}$ and Bioscience ${ }^{b}$ \\ University of Birmingham, Birmingham, United Kingdom \\ http://www.cs.bham.ac.uk/ axs/ \\ http://www.biosciences.bham.ac.uk/staff/staff.htm?ID=90 \\ (Commentary on Eva Jablonka and Marion Lamb: Evolution in Four Dimensions: Genetic, \\ Epigenetic, Behavioral, and Symbolic Variation in the History of Life, MIT Press, 2005
}

[This is a pre-print version. To appear in BBS 30(4) 2007])

\begin{abstract}
:
Jablonka \& Lamb (J\&L) refer only implicitly to aspects of cognitive competence that preceded both evolution of human language and language learning in children. These aspects are important for evolution and development but need to be understood using the design-stance, which the book adopts only for molecular and genetic processes, not for behavioural and symbolic processes. Design-based analyses reveal more routes from genome to behaviour than J\&L seem to have considered. This both points to gaps in our understanding of evolution and epigenetic processes and may lead to possible ways of filling the gaps.
\end{abstract}

Jablonka \& Lamb's (J\&L's) book exposes many tangled connections between genome, behaviour, and environment, but it skims over gaps in our knowledge about the information-processing capabilities underlying observed behaviours - ignoring important mechanisms with epigenetic features. Much is said about the physical and chemical mechanisms involved in development, but behavioural competences are described mostly from the outside. Explaining the internal information processing requires the design stance (Dennett 1978).

External behaviours of many animals indicate that they have mechanisms concerned with internal symbolic competences, required for perceiving or acting in structured situations, including planning, predicting, identifying information gaps to be filled, formulating goals, executing plans, learning generalisations, and creatively combining different competences. We need to explain what these competences are, what mechanisms make them possible, how they develop in individuals, and how they evolved. Such competences (in humans and other animals) seem to presuppose something like internal symbolic languages with very specific properties.

When the variety of structurally different combinations of situations and goals rules out preconfigured responses, animals need the ability to represent and make inferences about existing and future configurations and changes; for example, configurations of a partially constructed nest made of interlocking twigs and the affordances (Gibson 1979) for inserting the next twig. This requires internal formalisms for representing structures and possible processes and for constructing, comparing, and planning, including selecting actions from branching collections of possible future sequences. Later, the animal has to produce the actions under the control of the representation. So action sequences linked to complex internal symbolic structures occurred before external linguistic behaviour evolved.

Animal behaviours demonstrating such competences include tool-related behaviours (Kacelnik et al. 2006) and the remarkable symbolic competences of the grey parrot Alex

1 In Behavioral \& Brain Sciences Journal, Vol 30 No 4.2007. Commentary on Jablonka and Lamb, 2005 
(Pepperberg 2004).

Our epigenetic hypothesis about how information-processing develops under the influence of the environment avoids two extreme theories; first, that all animal competences are somehow encoded separately in the genome, possibly in a large collection of innate modules, and second, that a small collection of general learning mechanisms (e.g., reinforcement learning) is genetically determined and that everything else is a result of applying those general learning processes.

Our "middle way" also synthesises two apparently opposed views expressed by Karmiloff-Smith (1994, p. 693), "Decades of developmental research were wasted, in my view, because the focus was entirely on lowering the age at which children could perform a task successfully, without concern for how they processed the information," and Neisser (1976, p. 8), "we may have been lavishing too much effort on hypothetical models of the mind and not enough on analysing the environment that the mind has been shaped to meet."

What an individual can learn often changes dramatically during its life, indicating a cascaded development of competences partly under the influence of the environment, including competences to acquire new competences (metacompetences), some of which are themselves the result of interaction of earlier metacompetences with the environment. We summarise this relationship in Figure 1, showing multiple routes from the genome to behaviours of various sorts, with competences at different levels of abstraction and different sorts of specificity developed in different ways at different stages. This implies that learning in some parts of the brain is delayed until others have acquired a layer of competences to build on. So if prefrontal lobes are associated with processes further to the right of the diagram, occurring only after many cycles of simpler development, we would expect prefrontal lobes to develop after low-level visual and motor control mechanisms. Evidence consistent with this conjecture has recently been reported in human infants by Gilmore et al. (2007).

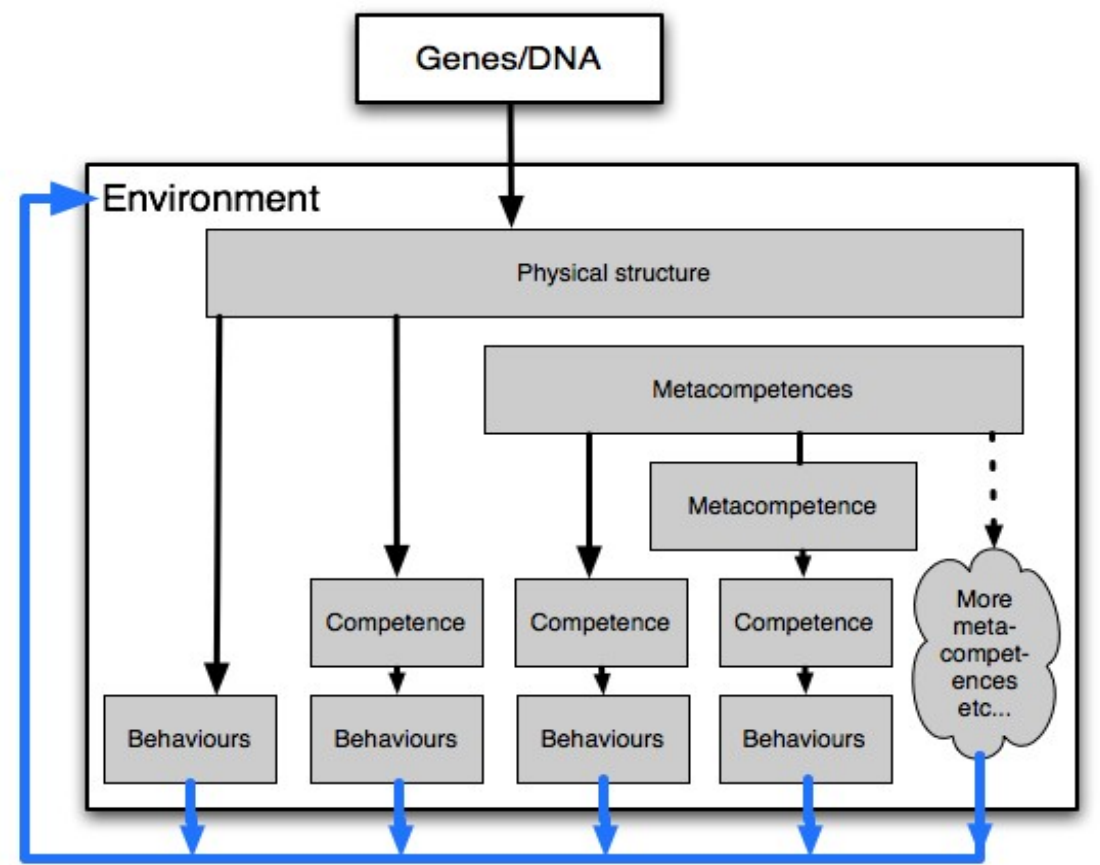

Figure 1 (Based on Chappell \& Sloman, 2007). The environment (including the body and new brain states) can affect all the processes. There are multiple routes from genome to behaviours, some of which are used only after others have produced new competences and metacompetences. 
J\&L discuss the evolution of language and, like many others (e.g., Arbib 2005), focus mainly on external language used for communication. This assumes that first there were simple forms of language (e.g., gestures and sounds), and complex forms evolved later.

In contrast, we suggest that language first evolved for "internal" use. Because some people restrict the label "language" to symbol systems used for external communication, we use the term g-language (generalised language) to refer to a wider class that includes internal languages. A g-language allows rich structural variability of various kinds as well as (context sensitive) compositional semantics for dealing with novel configurations of objects or processes.

Most people assume that language started simple and external and then grew more complex externally before being internalised. We, like Bridgeman (2005), suggest that complex g-languages evolved in many non-human species, and also develop in young children, who cannot yet talk. Internal g-languages are needed to provide forms of representation of current and possible future situations and processes that allow wide structural variation in what is represented, with compositional semantics to cope with novelty (Sloman 1979). So, rich internal g-languages are precursors to external human languages both in evolution and in child development. After g-languages had evolved for other purposes, including constructing plans that were used to control behaviour, some animals may have started mapping their internal structures onto external behaviours for communication purposes.

Insofar as animals and children can look at different parts of a scene and combine information from most recent saccades with information about parts of the scene that are no longer in view, for example, when planning what to do, they must use representations of spatial organisation of information as well as temporal organisation. In some ways, this requires more complex forms of representation than human spoken languages, combining aspects of verbal language and pictorial languages (analogous to maps, diagrams, and drawings; see also Trehub 1991).

G-languages probably evolved for internal information processing and control of behaviour (through the generation of goals, plans, or instructions), along with generation of questions to specify missing information, and perhaps to formulate hypotheses, explanations, and suppositions. External human language (spoken and gestural) and other symbolically based aspects of human culture (e.g., music, mathematics, and so on) also might have built on these preexisting internal symbolic foundations.

Eventually, instead of a specific g-language, evolution produced competences to acquire a variety of g-languages expressing different kinds of information. This implies that some nonhuman animals' behaviour will be directed and shaped by their internal glanguages, which in turn are shaped by the structure of the external environment, directing evolution down particular paths, and perhaps causing "convergent" evolution of closely related cognitive abilities in birds and mammals with overlapping perceptual and manipulative competences.

If abstract and complex g-language constructs have to be learnt at a late stage of development, but are particularly useful to a species, then some of them could become genetically assimilated or accommodated, in which case they will themselves become heritable and can direct development in particular ways. Environmental cues encountered by these animals will be filtered through their cognitive architecture, thus tightening the knots between the genome, the behaviour, and the environment. Chappell and Sloman (2007) suggest that this employed a separation between the parts of the mechanism producing a general class of behaviours and the parts that provide parameters that select 
from that class. The generic competence and the particular parameters might undergo separate trajectories in evolution and development.

If J\&L's "assimilate-stretch" principle were extended to cope with the evolution and development of internal g-languages and associated mechanisms, this might be a significant, previously unnoticed, factor in the evolution of cognition. Their examples, however, suggest that assimilate-stretch extends behaviour additively. But qualitatively new capabilities might emerge. For example, if a learned capability becomes genetically assimilated or accommodated, it could form a building block for qualitatively diverse competences. Information that some objects can be deformed by manipulation, can be broken into smaller pieces, can be inserted into spaces, and can, if appropriately assembled, produce fairly rigid structures, might form fundamental parts of a very complex collection of learnable competences, including constructing nests, making or using tools, or extracting objects from containers.

The ideas in this book may turn out to have far-reaching significance for many disciplines. We have tried to show, briefly, how some of that could affect studies of cognition, and internal g-languages, with implications for the evolution of language and many forms of learning. As our cited paper indicates, these forms of development may be required also for intelligent robots that are learning to cope in a wide variety of environments. $^{2}$

\section{Acknowledgements}

Some of this work arose out of discussions with colleagues in the EU-funded CoSy Robotic project at the University of Birmingham. Chris Miall helped with the diagram. Thanks to Erik Hollnagel for the Neisser quote.

\section{References}

Arbib, M. A. (2005). From monkey-like action recognition to human language: an evolutionary framework for neurolinguistics. Behavioral and Brain Sciences, 28(2):105--124. Web site: http://www.bbsonline.org/Preprints/Arbib-05012002

Bridgeman, B. (2005). Action planning supplements mirror systems in language evolution. Behavioral and Brain Sciences 28(2):129-130.

Chappell, J. M. \& Sloman A. (2007). Natural and artificial meta-configured altricial information-processing systems. International Journal of Unconventional Computing 3(3):211--239. Web Site: http://www.cs.bham.ac.uk/research/projects/cosy/papers/\#tr0609

Dennett, D. C. (1978). Brainstorms: philosophical essays on mind and psychology, MIT Press.

Gibson, J. J. (1979). The ecological approach to visual perception, Lawrence Erlbaum.

Gilmore J. H., Lin, W., Prastawa, M. W., Looney, C. B., Sampath, Y., Vetsa, K.,

2 These ideas are developed further on the Birmingham CoSy Project Web site: http://www.cs.bham.ac.uk/research/projects/cosy/papers 
Knickmeyer, R. C., Evans, D. D., Smith, J. K., Hamer, R. M., Lieberman, J. A. \& Gerig, G., (2007). Regional gray matter growth, sexual dimorphism, and cerebral asymmetry in the neonatal brain. The Journal of Neuroscience 27:1255-1260. Web site: doi:10.1523/JNEUROSCI.3339-06.2007

Jablonka, E. \& Lamb, M. J. (2005) Evolution in four dimensions: Genetic, epigenetic, behavioral, and symbolic variation in the history of life. MIT Press. Web site: http://www.bbsonline.org/Preprints/Jablonka-10132006/Referees/

Kacelnik, A., Chappell, J., Weir, A. A. S. \& Kenward, B. (2006). Cognitive adaptations for tool-related behaviour in New Caledonian crows, . In E. A. Wasserman \& T. R., E A and Zentall,, T R, (Eds) ., Comparative cognition: experimental explorations of animal intelligence, (pp. 515--528). Oxford: Oxford University Press Web site: http://www.cogsci.msu.edu/DSS/2004-2005/Kacelnik/Kacelnik_etal_Crows.pdf

Karmiloff-Smith, A. (1994). Precis of beyond modularity: a developmental perspective on cognitive science. Behavioral and Brain Sciences 17(4):693-745. Web site: http://www.bbsonline.org/documents/a/00/00/05/33/index.html

Neisser, U. (1976). Cognition and reality. W.H. Freeman.

Pepperberg, I. M. (2004) Lessons from cognitive ethology: Animal models for ethological computing. In: Proceedings of the First International Workshop on Epigenetic Robotics: Modeling Cognitive Development in Robotic Systems, Lund, Sweden, September 2001. Lund University Cognitive Studies, 85. Ed. C. Balkenius, J. Zlatev, H. Kozima, K. Dautenhahn, and C. Breazeal. Lund: LUCS. Web site: http://www.lucs.lu.se/ftp/pub/LUCS_Studies/LUCS85/Pepperberg.pdf

Sloman, A. (1979). The primacy of non-communicative language. In M. MacCafferty \& $\mathrm{K}$. Gray, Eds., The analysis of meaning: Informatics 5 Proceedings ASLIB/BCS Conference, Oxford, March 1979 (pp. 1-15). ASLIB. London. Web site: http://www.cs.bham.ac.uk/research/projects/cogaff/81-95.html\#43

Trehub, A. (1991). The cognitive brain, . Cambridge, MA: MIT Press,. 